\title{
Estudios en el género Cortaderia (Poaceae). II. Cortaderia ATACAMENSIS, NUEVO SINÓNIMO DE C. SPECIOSA
}

\author{
DANIEL TESTONI ${ }^{1}$
}

\begin{abstract}
Summary: Studies in the genus Cortaderia (Poaceae) II. Cortaderia atacamensis, a new synonym of $C$. speciosa. In this contribution to the systematics of the genus Cortaderia the synonymization of $C$. atacamensis and C. speciosa is proposed. Until now the binomial Cortaderia atacamensis has been considered a synonym of $C$. jubata. Nevertheless, the study of the type specimens and original descriptions, complemented with field studies, demonstrated that $C$. atacamensis is a synonym of $C$. speciosa (a species native to the desertic regions from Peru to northern Argentina and Chile), rather than of C. jubata (a species typical of the Andean forests from Colombia to northern Argentina). A morphological characterization and a key to separate the two species are provided.
\end{abstract}

Key words: Argentina, Chile, Cortaderia atacamensis, Cortaderia jubata, Cortaderia speciosa, Poaceae.

Resumen: En esta contribución a la sistemática del género Cortaderia se propone la sinonimización de C. atacamensis y C. speciosa. Hasta ahora el binomio Cortaderia atacamensis ha sido considerado un sinónimo de C. jubata. Sin embargo, el estudio de los ejemplares tipo y de las descripciones originales, complementado con estudios de campo, demostraron que C. atacamensis es un sinónimo de C. speciosa (una especie nativa de las regiones desérticas desde Perú hasta el norte de la Argentina y Chile), distinta de C. jubata (una especie típica de los bosques andinos desde Colombia hasta el norte de la Argentina). Se presentan una caracterización morfológica y una clave para separar ambas especies.

Palabras clave: Argentina, Chile, Cortaderia atacamensis, Cortaderia jubata, Cortaderia speciosa, Poaceae.

\section{INTRODUCCIÓN}

La sistemática interna de Cortaderia (Poaceae; Danthonioideae) -en particular de la sección Cortaderia- es confusa y, para poder establecer las relaciones filogenéticas entre sus especies, es necesario revisar integralmente el género (Linder et al., 2010).

Cortaderia atacamensis (Phil.) Pilg. y C. speciosa (Nees \& Meyen) Stapf son dos especies citadas para la puna argentina y el desierto chileno, cuya delimitación taxonómica no es clara: Acevedo de Vargas (1959) las considera como dos entidades diferenciables por la

\footnotetext{
${ }^{1}$ Herbario BBB, Departamento de Biología, Bioquímica y Farmacia, Universidad Nacional del Sur. San Juan 670, Bahía Blanca (8000), Argentina. Becario CONICET; daniel.testoni@uns.edu.ar
}

forma y color de la panoja; Conert (1961) las separa principalmente por el color de la nervadura central de la lema. Connor \& Edgar (1974) las tratan como dos especies muy afines, pero no las caracterizan morfológicamente, y restringen $C$. atacamensis a la localidad típica (San Pedro de Atacama, Chile). Astegiano et al. (1995) reconocen la afinidad de estos taxones y estiman que " $C$. atacamensis sería una forma de $C$. speciosa con espiguillas menores; pero consideramos aún prematuro proponer la sinonimia entre ambas entidades debido al escaso material disponible tanto en colecciones procedentes de Chile como de Argentina. De este modo, y al menos hasta que nuevas colecciones demuestren lo contrario, se restringe el área de $C$. atacamensis a la Puna de Chile". Romanutti et al. (2012) tratan a $C$. atacamensis como sinónimo de C. jubata (Lemoine ex Carrière) Stapf. 


\section{Antecedentes nomenclaturales}

Meyen (1834) utiliza dos nombres para designar colecciones provenientes de Nantoco (Chile): Gynerium neesii y G. speciosum, pero la publicación carece de descripciones. Posteriormente Nees \& Meyen -en una publicación de Nees (1843)incluyen ambos taxones bajo el nombre $G$. speciosum y describen la especie en base al ejemplar Meyen s/n "Ad flumen Copiapó dictum circa Nantoco in provincia Copiapó reipublicae Chilensis, Martio 1831". Este ejemplar es designado como lectotipo por Connor \& Edgar (1974) y se encuentra depositado en B.

Philippi (1865) describe Gynerium atacamense en base a un material juntado por él "Prope San Pedro de Atacama legit potest", cuyo holotipo se encuentra depositado en SGO.

Stapf (1897) es quien crea el género Cortaderia $\mathrm{y}$ hace el primer estudio taxonómico de las especies actualmente incluidas en la sección Cortaderia. A este género transfiere Gynerium argenteum Nees, G. quila Nees \& Meyen y G. speciosum Nees \& Meyen y describe dos nuevas especies: $C$. araucana y C. rudiuscula. A la especie de Atacama-Gynerium atacamense- la conoce por su descripción y no la incluye en su protólogo. Posteriormente Pilger (1906) la transfiere a Cortaderia.

\section{Material y Método}

Se estudiaron los ejemplares tipo y las colecciones de los herbarios BA, BAA, BAB, BBB, CHR, CONC, CORD, DAV, MVFA, SI y SGO (Thiers, 2014). Cuando no se dispuso del ejemplar tipo, se utilizó la foto del ejemplar, disponible en JSTOR (http://plants. jstor.org) o en las bases de datos de los herbarios.

Se revisó críticamente la nomenclatura de Cortaderia atacamensis y C. speciosa, y sus sinónimos (Index Kewensis, 1997; IPNI, 2010), según las normas establecidas en el Código Internacional de Nomenclatura Botánica (McNeill et al., 2012).

Se analizó la variabilidad interpoblacional e intrapoblacional de Cortaderia atacamensis y $C$. speciosa en base a los caracteres morfológicos reproductivos que han sido utilizados para caracterizar ambas especies: color, forma y longitud total de la panoja y de la rama basal, y la morfología de la espícula y del antecio basal. Para ello se visitaron 14 poblaciones: dos de C.atacamensis y $12 \mathrm{de}$ C. speciosa, que representan gran parte de la distribución conocida de ambas especies, incluyendo las localidades típicas. En cada una se muestrearon 10 individuos al azar y se analizaron 10 espículas de cada uno de ellos.

Poblaciones analizadas. Cortaderia atacamensis: Testoni 645 y 646; C. speciosa: Testoni 399, 404, 406, 407, 408, 410, 412, 413, 642, 643, 644 у 647. Los sitios se detallan en la sección "materiales examinados" y se indican con un asterisco $(*)$

\section{Resultados y Discusión}

Acevedo de Vargas (1959), en su revisión de las cortaderas chilenas, separa en la clave Cortaderia atacamenis de C. speciosa por presentar panojas laxifloras o densifloras respectivamente. Sin embargo, de la lectura crítica de este trabajo se extrae que ambas especies son descriptas con panojas densifloras, y es así como se observan en los ejemplares tipo y en las poblaciones originales visitadas. Además, en ese trabajo, las descripciones de las espículas de estas especies son casi idénticas.

Cortaderia atacamensis es una especie actualmente restringida a su localidad tipo, San Pedro de Atacama, Chile (Connor \& Edgard, 1974; Astegiano et al., 1995). La comparación de un gran número de espículas, coleccionadas en dos poblaciones de este sitio, y otras 12 poblaciones de $C$. speciosa, demostraron que son morfológicamente indiferenciables.

Estos resultados coinciden con los de Astegiano et al. (1995), quienes señalan, de manera preliminar, que Cortaderia atacamensis debería ser tratada como una variedad de $C$. speciosa por presentar antecios de menor tamaño. No obstante, el estudio de la variabilidad de C. speciosa permitió determinar que ambos taxones se solapan en todas las medidas de los caracteres usados tradicionalmente para diferenciarlos, lo que impide considerarlos como entidades distintas. Además, las poblaciones consideradas previamente como pertenecientes a ambas especies coinciden en su distribución geográfica, ecología y biología reproductiva: forman poblaciones muy homogéneas en márgenes de cuerpos de agua en las zonas desérticas andinas, donde únicamente el pie femenino está presente y su reproducción es únicamente asexual (apomixis).

La comparación de los ejemplares tipo confirman que ambos binomios corresponden a una única especie 
que, por el principio de prioridad nomenclatural, debe ser llamada Cortaderia speciosa (Nees \& Meyen) Stapf.

\section{Tratamiento taxonómico}

Cortaderia speciosa (Nees \& Meyen) Stapf, Gard. Chron. Ser. 3. Vol. 22: 396. 1897. Gynerium speciosum Nees \& Meyen, Nova Acta Leop. 19(1): 153. 1843. Moorea speciosa (Nees \& Meyen) Stapf, Gard. Chron. II. 400. 1903. Tipo: Chile, "Ad flumen Copiapo dictum circa Nantoco in provincia Copiapó reipublicae Chilensis", III-1831, F. J. F. Meyen s/n (Lectotipo B imagen!, designado por Connor \& Edgar, Taxon 23(4): 603. 1974, Isotipo BAA 3284!).

Gynerium quila Nees \& Meyen, Nova Acta Leop. 19(1): 153. 1843. Tipo: Chile, "In reipublica Chilensi ad Copiapó fluvium circa Nantoco", sine data, F. J. F. Meyen s/n (Sintipo B imagen!); Perú, "in Peruvia ad lacum Titicacam et ad pedem vulcani Arequipensis, Maio. Femina planta. Mascula ignota est.", 1000 m, sine data, F. J. F. Meyen s/n (Sintipo B imagen!; Isosintipo BAA!).

Gynerium quila Nees \& Meyen var. pygmaeum Meyen, Nova Acta Leop. 19(1): 153. 1843. Tipo: Perú, “ad lacum Titicacam. o", IV-1841, F. J. F. Meyen s/n (Holotipo B imagen!).

Gynerium atacamense Phil., Linnaea 33: 289. 1865. syn. nov. Cortaderia atacamensis (Phil.) Pilg., Bot. Jahrb. 37: 374. 1906. Tipo: Chile "Prope S. Pedro de Atacama", I-1854, R. A. Philippi s/n (Holotipo SGO 063853 imagen!; Isotipo BAA 2089!).

Gynerium argenteum Nees var. strictum E. Desv., Fl. Chile. 6: 328. 1854. Tipo: Chile, "Río Copiapó, junto a Nantoco", sine data, F. J. F. Meyen $\mathrm{s} / \mathrm{n}$ (Lectotipo B, designado por Connor \& Edgar, Taxon 23(4): 603. 1974).

Gynerium argenteum Nees var. parviflorum E. Desv., Fl. Chile. 6: 328. 1854. Tipo: Chile, "Mal Paso, cordillera de Guanta, a la orilla de los arroyos; elevación de 2490 met., en donde forma copas apretadas de un metro y mas", C. Gay s/n (Holotipo P).

Cortaderia rudiuscula Stapf, Gard. Chron. Ser. 3, 22: 396. 1897. Tipo. Chile, "Santa Rosa de los Andes", V-1882, Ball s.n. (Lectotipo K imagen!, designado por Connor \& Edgar, Taxon 23(4): 601. 1974; Isotipo BAA 1699!).

Iconografia. Acevedo de Vargas, 1959: fig. 2, fig. 4.; Romanutti et al., 2012: pág. 226.

Hierba perenne, cespitosa, robusta, en matas hemisféricas 1,5-3 m alt., con follaje abundante. Plantas únicamente pistiladas, apomícticas; se desconoce el pie estaminado. Hojas con vaina abierta, pilosa; lígula pilosa 1-2 mm long.; lámina plana de bordes cortantes, hasta $2 \mathrm{~m}$ long. Cañas floríferas que superan poco el follaje, a veces incluidas en él, muy numerosas en cada planta. Panojas 40-70 cm long., erectas, fusiformes, muy compactas, generalmente de color terroso, más raramente blancuzcas o con tintes purpúreos; ramas ascendentes, cortas, las inferiores 10 a 15 cm long. Espículas 8-15 mm long., (3) 4-6 floras, con o sin antecio superior rudimentario; glumas subiguales 6-8 mm long., generalmente menores que el antecio basal, enteras o bífidas en el ápice, uninerves, con nervio coloreado o no; lemas trinerves, con nervios coloreados o no, estrechándose gradualmente hacia el ápice y terminadas en una arista. Antecios con callo piloso, el basal con lema 7-12,5 mm long. (incluyendo la arista), con pelos rectos de 4 a $7 \mathrm{~mm}$, y con arista frágil $(0,5) 1-4 \mathrm{~mm}$ long., escabrosa, difícil de diferenciar del cuerpo de la lema, que a veces nace entre dos arístulas; pálea que alcanza la mitad del tamaño de su lema, con dos carinas evidentes; lodículas cuneadas, ciliadas; estaminodios 0,1-0,5 mm long., incluidos o no entre las lodículas. Ovario pequeño, estigmas exertos de 1,5 mm. Cariopsis 1,5 mm long., elongado-fusiforme, color pardo oscuro a la madurez.

Distribución y hábitat. Argentina, Chile, Bolivia y Perú, formando poblaciones homogéneas en borde de ríos de las zonas áridas desde el altiplano peruano hasta Mendoza, hasta los 4000 ms.m.

Material adicional examinado. ARGENTINA. Prov. Catamarca: Dpto. Antofagasta, vega La Cortadera (3600 ms.m.), 25-II-1995, Ruthsatz 9418 (SI); Antofagasta de la Sierra $\left(25^{\circ} 55^{\prime} 11^{\prime \prime}\right.$ S 6651'04"W, 3600 ms.m.), 20-III-2011, Testoni \& Villamil 412 (BBB)*; El Peñón (26²9’12”S 67¹9'56”'W, 3200 ms.m.), 21-III-2011, Testoni \& Villamil 413 (BBB)*. Prov. Jujuy: Dpto. Cochinoca, Cochinoca (2246'51'S 6600'30"W, 3600 ms.m.), 17-III-2011, Testoni \& Villamil 404 (BBB)*. Dpto. Humahuaca, Humahuaca (2931 ms.m.), sin fecha, Hunziker A. T. 1304 (BAA); Chapui Rodeo, 24III-1989, Novara et al. 8767 (SI, MCNS, CORD);

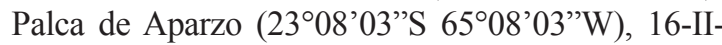
2009, Zuloaga et al. 9171 (SI); Hornaditas (2707'S $65^{\circ} 20^{\prime} \mathrm{W}$ ), 09-II-1979, Arenas \& Giberti s/n (SI, 
BACF 1020).Dpto. La Riconada, Rinconada (22²6’S 6609'W), 26-II-1980, Arenas \& Dell'Arciprete s/n (SI, BACAF 1880). Dpto. Santa Catalina, El Angosto (2152'01'’S 66²'53”'W, 3500 ms.m.), 17-III-2011, Testoni \& Villamil 407 (BBB)*; Santa Catalina (2154'54'’S 6503'16”'W, 3800 ms.m.), 17- III-2011, Testoni \& Villamil 408 (BBB)*; Santa Catalina, 08II-1980, Arenas \& Dell'Arciprete s/n (SI, BACAF

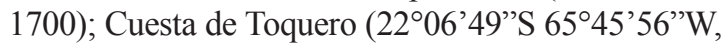
3600 ms.m.), 18-III-2011, Testoni \& Villamil 410 (BBB)*. Dpto. Susques, Susques, quebrada del Mal Paso (232 $5^{\prime} 55^{\prime}$ 'S $66^{\circ} 17^{\prime} 03^{\prime} \mathrm{W}, 3800$ ms.m.), 26-I2014, Testoni \& Montes 647 (BBB)*. Dpto. Tilcara, Garganta del Diablo, 28-II-1965, Fabris et al. 6003 (BAA); Maimará (2338'08'S 65²4'29”W, 2400 ms.m.), 15-III-2011, Testoni \& Villamil 399 (BBB)*. Dpto. Tumbaya, Cuesta de Quilpán $\left(23^{\circ} 41^{\prime} 51^{\prime}\right.$ S 6543'40”'W, 3800 ms.m.), 19-III-2011, Testoni \& Villamil 411 (BBB). Dpto. Yavi, Yavi Chico (2206'27'S 65²6’35”W; 3400 ms.m.), 17- III2011, Testoni \& Villamil 406 (BBB)*; Abra El Condor (22 53'51'S 65'17'17'W, 3600 ms.m.), 16-III-2011, Testoni \& Villamil 402 (BBB). Prov. La Rioja: Dpto. Gral. Lavalle, ca. Gualo, 25-I-1906, Kurtz 13268 (CORD). Dpto. Sanagasta, Sierra Vesasco, Yacuchi (2100 ms.m.), 28-II-1908, Kurtz 15491 (CORD). Dpto. Gral. Sarmiento, Cortaderas (3000 ms.m.), 01-II-1947, Hunziker J. H. 2110 (CORD). Dpto. Trancas, Sierra de Famatina, 13I-1947, Hunziker J. H. 1848 (BAA). Prov. Salta: Dpto. Cachi, Parque Nacional los Cardones (2514'S 6604"W, 2730 ms.m.), 14-II-2002, Ciadella et al. 266 (SI). Dpto. Los Andes, San Antonio de los Cobres, 29-I-1944, Cabrera 8259 (BAA). Prov. San Juan: Dpto. Calingasta, quebrada de Cortaderas (3050'11”S 69³4’32”W, 2800 ms.m.), 15-I-1999, Kiesling et al. 9115 (SI). Dpto. Sarmiento, sierra de Ansilta, quebrada Santa Clara (1950 ms.m.), 31-III1999, Biurrun et al. 5848 (SI). BOLIVIA. Dpto. La Paz, La Paz, 10-II-1919, Buchtien 507 (SI). Dpto. Oruro, Carangas, $11 \mathrm{~km}$ from Ancaraví towards Toledo (3850 ms.m.), 19-III-1999, Renvoize \& Flores 5247 (SI, CORD). Dpto. Potosi, Quechisla, XII-1931, Cárdenas 98 (SI). CHILE. Without locality, sin fecha, Gay s/n (BAA 3294, citado en Stapf, 1897); Andes of Chili, sin fecha, Cuming 386 (BAA, citado en Stapf, 1897). I Región Tarapacá: Prov. Arica, Arica, camino al Portezuelo de Chapiquiña (3450 ms.m.), 09-II-1964, Marticorena et al. 57 (MVFA, CONC), Prov. El Tamarugal, Pica (2130 ms.m.), III-1885,
Rahmer s/n (SGO 037220). Prov. Tarapacá, Aríaca, 1930, Elgueta 146 (SGO). II Región Antofagasta: Prov. El Loa, $78 \mathrm{~km} \mathrm{~N}$ of Calama (21'56'33'S 68`31'46”'W, 3300 ms.m.), 23-III-2001, Peterson \& Soreng 15609 (SI); Laguna Chiu Chiu, 23-XI-1941, Espinosa s/n (SGO 068539); San Pedro de Atacama (22॰53'52”S 6812'29”W, 2500 ms.m.), 25-I2014, Testoni \& Montes 645 (BBB, locus typicum de Gynerium atacamense Phil)*; ca. Puritama (22॰46’08'S 6804'30”W; 3200 ms.m.), 25-I-2014, Testoni \& Montes 646 (BBB)*. III Región Atacama: Prov. Copiapó, Nantoco, río Copiapó (27³2’05”S 70¹6’18”W, 550 ms.m.), 23-I-2014, Testoni \& Montes 644 (BBB, locus typicum de Gynerium speciosum Nees)*; idem, 26-I-1954, Acevedo de Vargas s/n (CONC 88092); Paipote, X-1956, Ricardi \& Marticorena 3644 (CONC). IV Región Coquimbo: Prov. Limarí, Talinay (3051'14'S 71³5'52'W, 300 ms.m.), 23-I-2014, Testoni \& Montes 643 (BBB)*. V Región Valparaíso: Prov. Los Andes, Santa Rosa de Los Andes, (32 51'39'S 70²5'36”W, 1100 ms.m.), 22-I-2014, Testoni \& Montes 642 (BBB, locus typicum de Cortaderia rudiuscula Stapf)*; Los Andes, I-1954, Acevedo de Vargas s/n (CONC 88084, isotopotipo de Cortaderia rudiuscula Stapf emend. Acevedo).

Obs. I. Los binomios: Gynerium neesii y $G$. pygmaeum, mencionados como especies nuevas por Meyen (1834), de Copiapó (Chile) y lago Titicaca (Perú) respectivamente, son sinónimos de Cortaderia speciosa, pero inválidos por carecer de descripciones (nom. nud.).

Obs. II. Conert (1961) designa como lectotipo de Gynerium atacamense Phil. el ejemplar Philippi 1024 (B imagen!), "Chile, oppidum Atacama, 1824". Sin embargo, el holotipo ha sido hallado en el herbario SGO (Connor, 1983).

Sobre la sinonimia de Cortaderia atacamensis con C. jubata:

Romanutti et al. (2012) consideran a Cortaderia atacamensis (considerado aquí como C. speciosa) sinónimo de C.jubata. Sin embargo estos taxones se diferencian claramente: $C$. jubata presenta panojas que emergen ampliamente del follaje, más grandes y llamativas, piramidales, amplias, laxas, con ramas laterales más largas y péndulas, de color purpúreo; glumas subiguales, del mismo largo que el antecio 


\section{Testoni - Cortaderia atacamensis, nuevo sinónimo de C. speciosa}

basal; lema largamente atenuada hacia el ápice, pero sin arista, con pelos sedosos, flexuosos, más largos; pálea que generalmente no supera un tercio de su lema. Habita en bosques y selvas húmedas desde el norte de la Argentina hasta Colombia y se comporta como invasora en Australia, Nueva Zelanda y oeste de los Estados Unidos.

Materiales de referencia. ECUADOR: Prov. Chimborazo, Chimborazo, 24-X-1895, Roezel s/n (Lectotypus K 307978 imagen!; Clastotypus BAA 1377!); entre Ríobamba y Alao (01 ${ }^{\circ} 48^{\prime} 10^{\prime \prime S}$ 78³5'26”'W; 2800 ms.m.), 06-IV-2012, Testoni \& Villamil 435 (BBB). Prov. Cotopaxi, at Laguna Quilotoa (051'S 7853'W, 3480 ms.m.), 19-II-1992, Lægaard 101349 (QCA, AAU). ESTADOS UNIDOS: Est. California, Contra Costa Co., along San Pablo Dam road, ca. 2 miles southeast of El Sobrante, 03-IX1959, Crampton 5456 (DAV). NUEVA ZELANDA: Auckland, Clifton Colliery, Kopuku, Waikato, 11-III1964, Connor s/n (CHR 148500).

Clave para diferenciar Cortaderia speciosa y $C$. jubata

A. Panoja fusiforme, muy compacta, con ramas rígidas, ascendentes y cortas (las inferiores de 15 $\mathrm{cm}$ ); lema aristada, con pelos rígidos, menores de $7 \mathrm{~mm}$ long..................Cortaderia speciosa

A'. Panoja piramidal, laxas, con ramas flexuosas, péndulas y largas (las inferiores mayores de 15 $\mathrm{cm}$ ); lema mútica, con pelos sedosos, mayores de $8 \mathrm{~mm}$ long. ..Cortaderia jubata

\section{Agradecimientos}

A los curadores de los herbarios, al CONICET, a Carlos B. Villamil y a dos revisores anónimos por sus valiosas sugerencias.

\section{Bibliografía}

ACEVEDO DE VARGAS, R. 1959. Las especies de Gramineae del género Cortaderia en Chile. Bol. Mus. Nac. Hist. Nat. Santiago de Chile 27: 205-246.

ASTEGIANO, M. E., A. M. ANTON \& H. E. CONNOR. 1995. Sinopsis del género Cortaderia (Poaceae) en la Argentina. Darwiniana 33: 43-51.
CONERT, H. J. 1961. Die systematik und anatomie der Arundineae. Weinheim: J. Cramer.

CONNOR, H. E. 1983. Names and types in Cortaderia Stapf (Gramineae) II. Taxon 32: 633-634.

CONNOR, H. E. \& E. EDGAR. 1974. Names and types in Cortaderia Stapf (Gramineae). Taxon 23: 595-605.

INDEX KEWENSIS. 1997. On compact disc version 2.0 for the IBM PC. United Kingdom: Oxford University Press.

IPNI, THE INTERNATIONAL PLANT NAMES INDEX. 2010. Published on the Internet http://www.ipni.org [acceso 2014].

LINDER, H. P., M. BAEZA, N. P. BARKER, C. GALLEY, A. M. HUMPHREYS, K. M. LLOYD, D. A. ORLOVICH, M. D. PIRIE, B. K. SIMON, N. WALSH \& G. A. VERBOOM. 2010. A generic classification of the Danthonioideae (Poaceae). Ann. Missouri Bot. Gard. 97: 306-364.

MCNEILL, J., F. R. BARRIE, W. R. BUCK, V. DEMOULIN, W. GREUTER, D. L. HAWKSWORTH, P. S. HERENDEEN, S. KANPP, K. MARHHOLD, J. PRADO, W. F. PRUD'HOMME VAN REINE, G. F. SMITH, J. H. WIERSEMA \& N. J. TURLAND. 2012. International Code of Nomenclature for algae, fungi and plants (Melbourne Code): adopted by the Eighteenth International Botanical Congress Melbourne, Australia, July 2011. Regnum Veg. 154. Königstein: Koeltz Scientific Books.

MEYEN, F. J .F. 1834. Reise um die Erde I. Berlin: Conrad Feister.

NEES VON ESENBECK, T. F. L. 1843. Gramineae. En: MEYEN, F. J. F., Observationes botanicas in itinere circum terram institutas: opus posthumum, Sociorum Academiae Curis Suppletum. Nova Acta Leop. 19(1). Vratislaviae: Eduard Weber's Buchhandlung.

PHILIPPI, R. A. 1865. Plantarum novarum Chilensium Centuriae inclusis quibusdam Mendocinis et Patagonicis. Linnaea 33: 1-308.

PILGER, R. 1906. Gramineae andinae II. In: URBAN, I. (ed.), Plantae novae andinae imprimis Weberbauerianae I. Bot. Jahrb. Syst. 37: 373-381.

ROMANUTTI, A. A.; A. M. ANTON \& H. E. CONNOR. 2012. Cortaderia. En: ANTON, A. M. \& F. O. ZULOAGA (eds.), Flora Vascular de la Republica Argentina: vol. 3, tomo 1, pp. 220-226. Córdoba: Gráficamente Ediciones.

STAPF, O. 1897. The botanical history of the Uva, Pampas Grass and their allies. Gard. Chron. 3(22): 396.

THIERS, B. Index Herbariorum: a global directory of public herbaria and associated staff. New York Botanical Garden's Virtual Herbarium, http://sweetgum.nybg. org/ih [acceso 2014].

Recibido el 23 de junio de 2014, aceptado el 24 de octubre de 2015. 
